\title{
Notas sobre a implementação dos cursos em Planejamento e Gestão em Saúde e caracterização das Hemorredes estaduais
}

\author{
Mariluce Karla Bomfim de Souza \\ Márcia Teixeira Gurgel do Amaral \\ Bárbara de Jesus Simões \\ Jussara Cargnin Ferreira
}

\section{SciELO Books / SciELO Livros / SciELO Libros}

SOUZA, M.K.B., AMARAL, M.T.G., SIMÕES, B.J., and FERREIRA, J.C. Notas sobre a implementação dos cursos em Planejamento e Gestão em Saúde e caracterização das Hemorredes estaduais. In: SOUZA, M.K.B., comp. Planejamento e gestão em saúde: caminhos para o fortalecimento das hemorredes [online]. Salvador: EDUFBA, 2018, pp. 125-143. ISBN 978-85-232-2027-3.

https://doi.org/10.7476/9788523220273.0008.

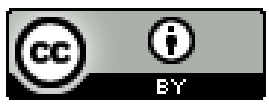

All the contents of this work, except where otherwise noted, is licensed under a Creative Commons Attribution 4.0 International license.

Todo o conteúdo deste trabalho, exceto quando houver ressalva, é publicado sob a licença Creative Commons Atribição $\underline{4.0}$. 


\title{
Notas sobre a implementação dos cursos em Planejamento e Gestão em Saúde e caracterização das Hemorredes estaduais ${ }^{1}$
}

\author{
Mariluce Karla Bomfim de Souza \\ Márcia Teixeira Gurgel do Amaral \\ Bárbara de Jesus Simões \\ Jussara Cargnin Ferreira
}

\section{Introdução}

O Projeto Planeja Sangue foi concebido por iniciativa da Coordenação Geral de Sangue e Hemoderivados do Ministério da Saúde (CGSH/MS) que visa apoiar as Hemorredes Estaduais, de maneira teórico-instrumental, para a concepção de Planos Diretores Estaduais de Sangue e Hemoderivados. Este plano é requisito legal na área de hematologia e hemoterapia, porém é limitado ao cotidiano das Hemorredes, devido à incipiente cultura institucional do planejamento nos serviços e pela complexidade encontrada para sua elaboração.

1 Agradecimentos: as autoras registram os agradecimentos aos gestores e/ou representantes das Hemorredes dos cinco estados: Nilza Sampaio e Aurora Cavalcanti (Bahia); Flora Sabino (Pernambuco); Sandra Sobreira (Paraíba); Gabrielle Duarte (Roraima); e, Alzira Saldanha (Mato Grosso) quanto à atualização das informações relacionadas à rede de serviços hemoterápicos. Agradecimento também a estudante Andréa Laís Santos e Santos pela atualização dos quadros relacionados à caracterização dos estados. 
As práticas de planejamento adotadas pela CGSH/MS, a partir do ano de 2008, foram norteadas pelo pressuposto da superação do planejamento normativo, envolvendo de forma sistemática a participação dos gestores da Hemorrede Pública Nacional e a força de trabalho da própria coordenação, consolidando ao longo do período, o uso de estratégias de fomento ao pensar coletivo e ampliado para o enfrentamento de situações-problema demandadas no âmbito do Sistema Nacional do Sangue.

No contexto citado, foi estabelecida cooperação técnica com o Instituto de Saúde Coletiva da Universidade Federal da Bahia (ISC/UFBA) visando o desenvolvimento de cursos de Atualização em Planejamento e Gestão de Sistema e Serviços de Saúde, que passou a ser denominado e conhecido como Planeja Sangue.

O desenvolvimento do Planeja Sangue para gestores e técnicos das hemorredes estaduais, ao longo do ano de 2015, permitiu a aproximação com a realidade dos estados, bem como o conhecimento sobre suas características demográficas, organização do sistema e distribuição dos serviços, de acordo com a divisão do estado em regiões de saúde, com especial atenção aos serviços de hematologia e hemoterapia.

Cada estado tem estabelecido, a partir de seu Plano Diretor de Regionalização da Saúde, o desenho destas regiões, de modo que a elaboração e o acompanhamento dos cursos permite conhecer as distintas realidades configuradas.

O presente capítulo contempla, por conseguinte, notas de registro sobre o processo de implementação do Planeja Sangue em Hemorredes estaduais do território nacional. Além disso, é apresentada uma breve caracterização destas hemorredes, para as quais foi ofertado o curso em Planejamento e Gestão no ano de 2015.

\section{Notas de registro sobre a implementação do Planeja Sangue}

A parceria e a articulação entre o Instituto de Saúde Coletiva da Universidade Federal da Bahia, reconhecido pela oferta/realização de cursos de graduação e pós-graduação e desenvolvimento de pesquisas, e a Coorde-

2 O curso de graduação em Saúde Coletiva, autorizado através da Portaria $n^{\circ} 813$, de 24 de agosto de 2007, do Ministério de Estado da Educação, DOU n 165 de 27.08.2007. (UNIVERSIDADE FEDERAL DA BAHIA, 2015) 
nação-Geral de Sangue e Hemoderivados do Ministério da Saúde, permitiu a implementação do Planeja Sangue na Hemorrede Pública Nacional, iniciando-se o trabalho, no ano de 2015, nos estados da Bahia, Roraima, Mato Grosso, Pernambuco e Paraíba.

A oferta dos cursos, após o aceite das Hemorredes Estaduais, se deu por meio de edital público para cada um dos estados citados, com o fomento à representação dos serviços de hematologia e hemoterapia dos diversos níveis de complexidade, obtendo-se a participação de profissionais e gestores dos Hemocentros Coordenadores (HC), Hemocentros Regionais (HR), Núcleos de Hemoterapia (NH), Unidades de Coleta e Transfusão (UCT), Agências Transfusionais (AT) e demais diretorias e coordenações estaduais como Planejamento, Atenção Básica (AB), Vigilância Sanitária (VISA) e Vigilância Epidemiológica (VE), além de organizações de representação de usuários e Conselho Estadual de Saúde, sendo registradas pequenas variações da participação destes segmentos nos estados.

A distribuição quantitativa ${ }^{3}$ dos participantes, bem como os municípios que representaram cada estado, pode ser observada a seguir:

- Bahia: 38 participantes de 20 municípios;

- Roraima: 48 participantes de 3 municípios;

- Mato Grosso: 53 participantes de 19 municípios;

- Pernambuco: 42 participantes de 09 municípios;

- Paraíba: 77 participantes de 17 municípios.

Pode-se observar a variação no número de participantes em cada estado, o que pressupõe motivos distintos, por exemplo: disponibilidade para a participação dos cursistas em todos os momentos presenciais; quantitativo de profissionais nos serviços da Hemorrede; extensão territorial do estado; agenda concorrida pelos compromissos profissionais e/ou pessoais, dentre outros. Quanto aos municípios que foram representados em cada estado,

3 Este quantitativo de participantes e de municípios refere-se aos dados coletados da lista de participantes do Momento presencial 1 (M1) e da lista de inscritos no curso por estado, respectivamente. 
pressupõe que a diferença pode ser atribuída à distribuição e localização dos serviços da Hemoterapia em cada território estadual.

No processo de implementação do Planeja Sangue, em 2015, foram registradas variadas observações de formato, de método e de logística levadas à discussão com a equipe técnica e de coordenação para ajustes imediatos, quando possíveis, e para discussão no Encontro de Avaliação agendado para o mês de novembro daquele ano.

No que tange ao formato da oferta do curso desenhado em três momentos presenciais (M1, M2, M3) e dois momentos de dispersão (D1, D2) foi possível observar sua aplicação no que havia sido proposto, contemplando o acesso e revisão às bases teóricas para o planejamento e a imersão na realidade, iluminados por tais conhecimentos para o desenvolvimento do processo de planejamento regional e desenho preliminar dos planos diretores da Hemorrede.

A execução do curso dentro do formato proposto foi possível pelos métodos utilizados. Para cada cursista foi disponibilizado um "Guia para estudos", de Souza e colaboradores (2015) e o cadastro para acesso a plataforma Moodle que possibilitou o espaço de interação entre cursistas e facilitadores durante os momentos de dispersão.

O arcabouço teórico apresentado no guia e discutido com os docentes nos momentos presenciais, bem como, a realidade problematizada em trabalhos de grupo e estudo de caso, esclareceu os cursistas sobre o desenvolvimento do processo de planejamento em saúde. Além disso, a análise da situação de saúde local proposta nos momentos M1 e D1 possibilitou a percepção ampliada sobre o seu estado e, a partir do desenho de objetivos, estratégias de ações e proposição de intervenções e análise da viabilidade, foi elaborada a proposta preliminar de Plano Diretor para a Hemorrede Estadual.

A variedade na composição das turmas, para além daqueles trabalhadores inseridos nos serviços de hemoterapia, era uma situação prevista e, por isso, o desenho do curso com os recursos e métodos propostos foi elaborado a fim de incluir e comprometer todos os participantes no (re)conhecimento do estado, dos serviços hemoterápicos e da atenção hematológica dentro do Sistema Único de Saúde.

No "Guia de Estudos" o capítulo Estudo de caso (2015) foi um dos recursos utilizados durante o momento presencial e que oportunizou o conhecimento daqueles cursistas que não atuam diretamente nos serviços de Hemo- 
terapia e o (re)conhecimento dos trabalhadores e gestores que lidam no seu cotidiano com as questões e os problemas relacionados à política do sangue, sejam eles nos serviços de atenção às pessoas com alguma doença hematológica, seja nos serviços hemoterápicos.

Quanto à logística para a realização dos cursos, para cada estado foi disponibilizada uma equipe constituída por: dois docentes e um técnico administrativo do ISC/UFBA; dois facilitadores técnicos atuantes nas diferentes Hemorredes estaduais; e, um mediador da CGSH/MS. No dia anterior ao início de cada momento presencial toda a equipe se deslocava para a capital do estado no qual o curso foi ofertado levando o material impresso (guias, declarações, etc.) e outros necessários para a sua operacionalização. Aos cursistas, também cabia tal deslocamento. Assim, providências de passagens e translados para a equipe, bem como, alimentação e hospedagem para equipe e cursistas faziam parte da agenda de atividades para a execução dos cursos.

Portanto, todas as notas de registro sobre sua implementação, especificamente sobre formato, método e logística foram discutidas no Encontro de Avaliação do Planeja Sangue que aconteceu em Salvador nos dias 27 e 28 de novembro de 2015 com a participação dos coordenadores institucionais, mediadores, facilitadores e docentes. Este encontro marcou o encerramento da agenda do "Planeja Sangue" de 2015 e representou importante momento de avaliação das ações e atividades implementadas durante o ano. Em síntese, foi apontado pelos presentes a eficácia do método utilizado, contudo, evidenciou-se a necessidade de aperfeiçoamento na operacionalização de questões de logística e também pequenos ajustes na distribuição e carga horária dos conteúdos, entretanto, sem modificação do formato geral da proposta.

Ressalta-se, que a etapa de avaliação permeou todos os momentos presenciais e de dispersão dos cursos e direcionou o trabalho realizado pelo grupo coordenador durante a execução dos mesmos. A "avaliação" implica no posicionamento positivo ou negativo em relação ao objeto, ato ou curso da ação que está sob julgamento, requerendo, portanto, uma tomada de posição favorável ou desfavorável ao que está sendo avaliado, logo, uma decisão de ação. (LUCKESI, 2003)

Diante das experiências vivenciadas e conclusão da oferta de cinco cursos, os resultados e impactos só poderão ser aferidos em médio e longo prazo, visto que a institucionalização das práticas de planejamento requer 
mudanças nas pessoas e nas organizações e que a proposta de Plano Diretor desenhada preliminarmente no decorrer dos cursos deverá ser finalizada e, portanto, legitimada nas instâncias formais do Sistema Único de Saúde. No entanto, acredita-se que sem um instrumento formal de planejamento não há como pautar a área de sangue como prioridade da agenda governamental, daí a relevância do Planeja Sangue como instrumento de qualificação da gestão da Hemorrede pública nacional.

\section{Caracterização das Hemorredes Estaduais}

Para a organização das Hemorredes Estaduais, a distribuição dos serviços de hematologia e hemoterapia deve seguir a regulamentação contida na Portaria/GM n ${ }^{\circ} 1.631$, de $1^{\circ}$ de outubro de 2015, provendo essa assistência aos usuários do Sistema Único de Saúde. Ressalta-se, que a divisão por macrorregiões e regiões de saúde deve assegurar atendimento às necessidades dos munícipes que delas fazem parte, principalmente no que se refere às ações de média densidade tecnológica, caracterizando-se como referência, nesse nível de atenção à saúde, para a população local.

De acordo com a Lei $n^{\circ} 10.205 / 2001$, cabem aos estados, além do Distrito Federal e municípios, por meio de suas secretarias de saúde ou equivalentes, a coordenação e execução das ações correspondentes a Política de Sangue, Componentes e Hemoderivados no seu âmbito de atuação, em articulação com o Ministério da Saúde. (BRASIL, 2001) No entanto, historicamente, o desenho e a implantação dos hemocentros coordenadores datam de períodos anteriores a lei.

O Projeto Planeja Sangue, em 2015, ofertou cursos voltados para planejamento e gestão de hemorredes em cinco estados, a saber: Bahia (BA); Pernambuco (PE); Paraíba (PB); Roraima (RR); e, Mato Grosso (MT); contemplando, portanto, três regiões geográficas brasileiras - Nordeste, Norte e Centro-Oeste, respectivamente.

A seguir apresenta-se uma breve caracterização das hemorredes nas quais os cursos foram ofertados.

\section{I) Hemorrede do estado da Bahia}

A Bahia, maior estado do nordeste brasileiro em termos demográficos, com população que ultrapassa 15 milhões de habitantes (IBGE, [201-]), con- 
centra aproximadamente $7,3 \%$ do total de habitantes do país. (BAHIA, 2012b) Dos 417 municípios, cerca de $60 \%$ possui menos de 20.000 habitantes, e, aproximadamente, $30 \%$ da população do estado reside em zona rural. (IBGE, 2011)

O quadro abaixo mostra dados sobre o perfil do estado que permitem conhecer suas características, inclusive sobre renda per capita, esperança de vida ao nascer, mortalidade infantil, coletas de sangue e taxa de doação de sangue.

Quadro 1 - Perfil estadual por área, população, renda per capita, esperança de vida ao nascer, mortalidade infantil, coleta e taxa de doação de sangue, Bahia, 2016

\begin{tabular}{|c|c|}
\hline Superfície territorial ${ }^{[1]}$ & $564.733,081 \mathrm{~km}^{2}$ \\
\hline População ${ }^{[1]}$ & 15.203.934de habitantes \\
\hline Rendimento per capita da população residente (reais) ${ }^{[1]}$ & $R \$ 736,00$ \\
\hline Esperança de vida ao nascer [2] & $\begin{array}{l}67,7 \text { anos (homens) } \\
76,4 \text { anos (mulheres) }\end{array}$ \\
\hline Mortalidade infantil (3) & 20,1/1.000 nascidos vivos \\
\hline Coleta de sangue anual ${ }^{(4)}$ & 208.181 coletas \\
\hline Taxa de doação ${ }^{(4)}$ & 13.76/1.000 habitantes \\
\hline
\end{tabular}

(1) e (2) IBGE ([2O1-]); (3) Brasil (2012); (4) Brasil (2015).

Fonte: elaborado pelos autores com base em IBGE ([201-]) e Brasil (2012, 2015).

Segundo o Plano Diretor de Regionalização da Saúde, a Bahia está dividida em 9 macrorregiões e 28 regiões ${ }^{4}$ de saúde, no qual encontram-se distribuídos os serviços, de acordo com a oferta disponibilizada pelos municípios e a capacidade instalada.

Em dezembro de 2014, a Lei Estadual nº 13.204 determinou a extinção das Diretorias Regionais de Saúde (Dires) e a criação dos Núcleos Regionais de Saúde (NRS) que têm por finalidade acompanhar as atividades de regulação e vigilância sanitária, bem como ações relativas à Coordenação de Monitoramento de Prestação de Serviços de Saúde; à Central de Aquisições e Contratações da Saúde; e à Corregedoria da Saúde, contribuindo para o fortalecimento da gestão junto aos Municípios. (BAHIA, 2014)

4 As regiões de saúde do estado da Bahia estão definidas na Resolução da CIB nº 275/2012. (BAHIA, 2012a) 
$\mathrm{O}$ atendimento às demandas de sangue e hemocomponentes da rede pública, em Salvador e região metropolitana, teve início em janeiro de 1983, em uma área adaptada do Hospital Geral Roberto Santos. Em 26 de julho de 1989, foi criada a Fundação de Hematologia e Hemoterapia da Bahia (Hemoba), tendo por finalidade exercer atividades de hematologia e hemoterapia em todo Estado, inclusive o desenvolvimento de ensino e pesquisa. Em 1992, a Fundação Hemoba passou a funcionar em espaço próprio e atualmente, tem unidades hemoterápicas distribuídas em diversas regiões do estado. (FUNDAÇÃO DE HEMATOLOGIA E HEMOTERAPIA DO ESTADO DA BAHIA, 2016)

O Quadro 2 mostra a distribuição das unidades de assistência hemoterápica, classificadas de acordo com a Resolução da Diretoria Colegiada (RDC) da Anvisa, $n^{\circ} 151$, de 21 de agosto de 2001, existentes no estado e que compõem a rede Hemoba.

Quadro 2 - Distribuição dos serviços de hematologia e hemoterapia do Sistema Único de Saúde nas macrorregiões, Bahia, 2016

\begin{tabular}{|c|c|}
\hline Macrorregiões & Quantidade/Unidades por nível de complexidade/municípios \\
\hline Centro Leste & OЗ UCT (Feira de Santana; Itaberaba; Seabra) \\
\hline Centro-Norte & Oટ UCT (Irecê; Jacobina) \\
\hline \multirow[t]{2}{*}{ Extremo Sul } & Ol HR (Eunápolis) \\
\hline & Ol UCT (Teixeira de Freitas) \\
\hline \multirow[t]{3}{*}{ Leste* $^{*}$} & $\mathrm{Ol} \mathrm{HC} \mathrm{(Salvador)}$ \\
\hline & O3 UC (O1 UC SAC/Cajazeiras; Ol UC Hospital Santo Antônio; Ol UC Subúrbio) \\
\hline & Oટ UCT (Camaçari; Santo Antônio de Jesus) \\
\hline Nordeste & Oટ UCT (Alagoinhas; Ribeira do Pombal) \\
\hline Norte & O3 UCT (Juazeiro; Paulo Afonso; Senhor do Bonfim) \\
\hline Oeste & Ol UCT (Barreiras) \\
\hline Sudoeste & O4 UCT (Brumado; Guanambi; Itapetinga; Vitória da Conquista) \\
\hline \multirow[t]{2}{*}{ Sul } & Ol UC (Valença) \\
\hline & Ol UCT (Jequié) \\
\hline
\end{tabular}

* Na Macrorregião Leste, estão disponíveis O2 UC - Hemóvel (veículo para realização de coletas externas).

Fonte: Fundação de Hematologia e Hemoterapia do Estado da Bahia (2016). 
A divisão por macrorregiões e regiões de saúde deve ser no sentido de garantir atendimento às necessidades dos munícipes que delas fazem parte, principalmente no que se refere às ações de média densidade tecnológica, caracterizando-se como referência nesse nível de atenção à saúde para a população local e apresentando algum grau de resolutividade. Essa mesma lógica deveria corresponder à distribuição dos serviços para atenção hematológica e também dos serviços hemoterápicos.

\section{II) Hemorrede do estado de Pernambuco}

Também na região Nordeste, o curso foi ofertado ao estado de Pernambuco. A população do estado é estimada em mais de nove milhões de habitantes (IBGE, [201-]), distribuídos em 184 municípios e um território estadual - Fernando de Noronha -, sendo, pouco mais de $80 \%$, considerados municípios de pequeno porte. (PERNAMBUCO, 2012)

Outras informações sobre superfície territorial, renda per capita, esperança de vida ao nascer, mortalidade infantil, coletas e taxa de doação de sangue, possibilitam caracterizar mais amplamente o estado de Pernambuco, conforme mostra o quadro a seguir.

Quadro 3 - Perfil estadual por área, população, renda per capita, esperança de vida ao nascer, mortalidade infantil, coleta e taxa de doação de sangue, Pernambuco, 2016

\begin{tabular}{|l|l|}
\hline Superfície territorial ${ }^{[1]}$ & $98.076,109 \mathrm{~km}^{2}$ \\
\hline População $^{[1]}$ & 9.345 .173 de habitantes \\
\hline Rendimento per capita da população residente (reais) [1] & $R \$ 822,00$ \\
\hline Esperança de vida ao nascer [2] $^{[2]}$ & $\begin{array}{l}66,8 \text { anos (homens] } \\
75,5 \text { anos (mulheres) }\end{array}$ \\
\hline Mortalidade infantil $^{[3]}$ & $15,6 / 1.000$ nascidos vivos \\
\hline Coleta de sangue anual $^{[4]}$ & 224.564 coletas \\
\hline Taxa de doação ${ }^{(4)}$ & $24,20 / 1.000$ habitantes \\
\hline
\end{tabular}

(1) e (2) IBGE ([2O1-]); (3) Brasil (2O12); (4) Brasil (2O15).

Fonte: elaborado pelos autores com base em IBGE ([201-]] e Brasil (2012, 2015].

Pernambuco está dividido em quatro macrorregiões e 12 regiões de saúde, conforme estratégia de regionalização e em consonância com o PDR/PE. 
No âmbito técnico administrativo, as regiões estão sob a coordenação das respectivas Gerências Regionais de Saúde (Geres) que têm a missão de planejar, executar, acompanhar, monitorar e avaliar as ações e serviços e elaborar as diretrizes da política estadual em cada região de saúde. (PERNAMBUCO, 2011, 2012)

O Hemocentro de Pernambuco (Hemope) foi o primeiro a ser implantado no Brasil, fundado em 25 de novembro de 1977. Posteriormente, por força de lei complementar em 1990, o Hemope recebeu nova denominação, enquanto entidade dotada de personalidade jurídica de direito público, de caráter científico, tecnológico, educacional e de prestação de serviços, sem fins lucrativos, vinculada à Secretaria de Saúde do Estado (BRASIL, 2010), por meio de administração indireta fundacional.

Atualmente, a Hemorrede Estadual de Pernambuco dispõe da seguinte distribuição de serviços, por macrorregiões de saúde.

Quadro 4 - Distribuição dos serviços de hematologia e hemoterapia do Sistema Único de Saúde nas macrorregiões, Pernambuco, 2016

\begin{tabular}{|c|c|}
\hline Macrorregiões [e regiões] & Quantidade/Unidades por nível de complexidade/municípios \\
\hline \multirow{4}{*}{$\begin{array}{l}\text { I - Metropolitana } \\
\text { (regiões I, II, III e XII] }\end{array}$} & $\mathrm{Ol} \mathrm{HC} \mathrm{(Recife)}$ \\
\hline & Ol UCT (Recife) \\
\hline & O己 AT Regional (Palmares; Limoeiro) \\
\hline & O4 AT (Recife; Jaboatão dos Guararapes; Cabo de Santo Agostinho; Tamandaré) \\
\hline \multirow{2}{*}{$\begin{array}{l}\text { II-Agreste } \\
\text { (regiões IV e V) }\end{array}$} & O己 HR (Caruaru; Garanhuns) \\
\hline & Oટ AT (Petrolândia; Caruaru) \\
\hline \multirow{3}{*}{$\begin{array}{l}\text { III - Sertão } \\
\text { (regiões VI, Xe XI) }\end{array}$} & Ol HR (Serra Talhada) \\
\hline & OI NHR (Arcoverde) \\
\hline & Ol AT (Quixaba) \\
\hline \multirow{3}{*}{$\begin{array}{l}\text { IV - Vale do São Francisco e } \\
\text { Araripe } \\
\text { (regiões VII, VIII e IX] }\end{array}$} & OI NHR (Salgueiro) \\
\hline & O己 HR (Petrolina; Ouricuri) \\
\hline & O3 AT (Araripina; Cabrobó; Belém do São Francisco) \\
\hline
\end{tabular}

Fonte: Fundação Hemocentro de Pernambuco (2016). 


\section{III] Hemorrede Estadual da Paraíba}

Outro estado do Nordeste brasileiro, para o qual o curso foi ofertado, foi a Paraíba. O referido estado é composto por 223 municípios, com população estimada em 2015 de aproximadamente 4 milhões de habitantes. (IBGE, [201-])

O estado da Paraíba foi caracterizado, ainda, pelas informações de superfície territorial, renda per capita, esperança de vida ao nascer, mortalidade infantil, coletas e taxa de doação de sangue, apresentadas no quadro abaixo.

Quadro 5 - Perfil estadual por área, população, renda per capita, esperança de vida ao nascer, mortalidade infantil, coleta e taxa de doação de sangue, Paraíba, 2016

\begin{tabular}{|l|l|}
\hline Superfície territorial (1) & $56.469,744\left(\mathrm{~km}^{2}\right)$ \\
\hline População (1) & 3.972 .202 de habitantes \\
\hline Rendimento per capita da população residente (reais) (1) & R $\$ 776,00$ \\
\hline Esperança de vida ao nascer (2) & $\begin{array}{l}67,4 \text { anos (homens) } \\
75,0 \text { anos (mulheres) }\end{array}$ \\
\hline Mortalidade infantil (3) & $17,5 / 1.000$ nascidos vivos \\
\hline Coleta de sangue anual (4) & 80.338 coletas \\
\hline Taxa de doação (4) & $20,37 / 1.000$ habitantes \\
\hline
\end{tabular}

(1) e (2) IBGE ([2O1-]); (3) Brasil (2O12); (4) Brasil (2O15).

Fonte: elaborado pelos autores com base em IBGE ([201-]) e Brasil (2012, 2015).

O modelo de reorganização da saúde, no estado da Paraíba, foi estruturado pelo Núcleo de Desenvolvimento das Regiões, dentro da Gerência de Planejamento e Gestão, e pelas Gerências Regionais de Saúde, sob a justificativa de que "cada gerência regional conta com uma capacidade funcional instalada, capaz de articular o processo gerencial das ações técnico-administrativas da saúde". (PARAÍBA, 2008, p. 15)

Constituído por 4 macrorregiões e 16 regiões de saúde, o estado tem distribuído os serviços de hemoterapia, considerando as tipologias de serviços previstos na $\mathrm{RDC} \mathrm{n}^{\circ} 151 / 2001$, conforme quadro a seguir: 
Quadro 6 - Distribuição dos serviços de hematologia e hemoterapia do Sistema Único de Saúde nas macrorregiões, Paraíba, 2016

\begin{tabular}{|l|l|}
\hline Macrorregiões & Quantidade/Unidades por nível de complexidade/municípios \\
\hline \multirow{3}{*}{ João Pessoa } & Ol HC (João Pessoa) \\
\cline { 2 - 2 } & O2 NH (Guarabira, Itabaiana) \\
\cline { 2 - 2 } & 14 AT (João Pessoa) \\
\hline Campina Grande & O1 HR (Campina Grande) \\
\cline { 2 - 2 } & O2 NH (Picuí, Monteiro) \\
\cline { 2 - 3 } & O9 AT (Campina Grande) \\
\hline Patos & O4 NH (Patos, Itaporanga, Princesa Isabel, Piancó) \\
\hline Sousa & NH (Sousa, Cajazeiras, Catolé do Rocha) \\
\cline { 2 - 3 } & O1 AT (Pombal) \\
\hline
\end{tabular}

Fonte: Paraíba [2016].

O Hemocentro da Paraíba (Hemoíba) foi criado, por meio da Lei $n^{\circ} 6.306$ de 2 de fevereiro de 1991, para ser referência na assistência hemoterápica no estado. No entanto, a interiorização da Hemorrede ocorreu a partir de 1996, com o objetivo de atender a toda população, ficando distribuída nos municípios sede dos Núcleos Regionais de Saúde (NRS). Atualmente, a Hemorrede é a única fornecedora de produtos hemoterápicos da rede pública e em quase totalidade da rede privada de serviços de saúde. (BRASIL, 2010)

\section{IV) Hemorrede do estado de Roraima}

Com uma população estimada em pouco mais de meio milhão de habitantes em 2015, o estado de Roraima, da região Norte do Brasil, possui grande extensão territorial com baixa densidade demográfica, quando comparado aos demais estados onde o curso foi ofertado. A rede de serviços de saúde se distribui em 15 municípios, em duas macrorregiões, segundo o Plano Diretor de Regionalização, sendo a maior parte dos serviços localizados na capital do estado.

O estado de Roraima foi caracterizado, também, pelas informações de superfície territorial, renda per capita, esperança de vida ao nascer, mortalidade infantil, coletas e taxa de doação de sangue, apresentadas no quadro abaixo. 
Quadro 7 - Perfil estadual por área, população, renda per capita, esperança de vida ao nascer, mortalidade infantil, coleta e taxa de doação de sangue, Roraima, 2016

\begin{tabular}{|c|c|}
\hline Superfície territorial ${ }^{[1]}$ & $224.303,187\left(\mathrm{~km}^{2}\right)$ \\
\hline População ${ }^{[1]}$ & 505.665 de habitantes \\
\hline Rendimento per capita da população residente (reais) ${ }^{[1]}$ & $\mathrm{R} \$ 1.008,00$ \\
\hline Esperança de vida ao nascer ${ }^{[2]}$ & $\begin{array}{l}66,9 \text { anos (homens) } \\
72,5 \text { anos (mulheres) }\end{array}$ \\
\hline Mortalidade infantil (3) & $15,4 / 1.000$ nascidos vivos \\
\hline Coleta de sangue anual ${ }^{[4]}$ & 11.398 coletas \\
\hline Taxa de doação ${ }^{(4)}$ & 22,94/ 1.000 habitantes \\
\hline
\end{tabular}

(1) e (2) IBGE ([2O1-]); (3) Brasil (2012); (4) Brasil (2015).

Fonte: elaborado pelos autores com base em IBGE ([201-]) e Brasil (2012, 2015).

O Hemocentro de Roraima (Hemoraima) foi inaugurado em setembro de 1992 e está vinculado à Secretaria Estadual de Saúde de Roraima. Atualmente, a Hemorrede Estadual conta com serviços conforme a distribuição regional apresentada no quadro a seguir.

Quadro 8 - Distribuição dos serviços de hematologia e hemoterapia do Sistema Único de Saúde nas macrorregiões, Roraima, 2016

\begin{tabular}{|l|l|}
\hline Macrorregiões & Quantidade/Unidades por nível de complexidade/municípios \\
\hline Centro Norte & O1 HC (Boa Vista) \\
\cline { 2 - 2 } & O3 AT (Boa Vista) \\
\cline { 2 - 2 } & O4 NH (Boa Vista) \\
\hline Sul & O1 AT (Rorainópolis) \\
\hline
\end{tabular}

Fonte: elaborado pelas autoras.

\section{V) Hemorrede do estado de Mato Grosso}

A população urbana do estado de Mato Grosso triplicou de tamanho entre os anos de 1980 e 2010, sendo, em 2015, 3.265.486 habitantes, distribuídos em 141 municípios. (IBGE, [201-]; MATO GROSSO, 2013) 
Da mesma forma que os demais estados participantes do Planeja Sangue, o estado do Mato Grosso foi caracterizado pelas informações de superfície territorial, renda per capita, esperança de vida ao nascer, mortalidade infantil, coletas e taxa de doação de sangue, apresentadas no quadro abaixo.

Quadro 9 - Perfil estadual por área, população, renda per capita, esperança de vida ao nascer, mortalidade infantil, coleta e taxa de doação de sangue, Mato Grosso, 2016

\begin{tabular}{|c|c|}
\hline Superfície territorial ${ }^{[1]}$ & $903.378,292\left(\mathrm{~km}^{2}\right)$ \\
\hline População & 3.265.486 de habitantes \\
\hline Rendimento per capita da população residente (reais] (1]) & $R \$ 1.055,00$ \\
\hline Esperança de vida ao nascer (2) & $\begin{array}{l}69,5 \text { anos (homens) } \\
75,9 \text { anos (mulheres) }\end{array}$ \\
\hline Mortalidade infantil ${ }^{[3]}$ & 18,5/1.000 nascidos vivos \\
\hline Coleta de sangue anual ${ }^{[4]}$ & 80.759 coletas \\
\hline Taxa de doação ${ }^{[4]}$ & $25,05 / 1.000$ habitantes \\
\hline
\end{tabular}

(1) e (2) IBGE ([2O1-]); (3) Brasil (2012); (4) Brasil (2015).

Fonte: elaborado pelos autores com base em IBGE ([201-]) e Brasil (2012, 2015).

No âmbito da regionalização da saúde, o referido estado está organizado em 16 regiões de saúde, considerados espaços estratégicos para a gestão e desenvolvimento integrado das políticas de saúde. Cada região possui Escritório Regional de Saúde (ERS) que responde pela condução da política estadual de saúde, em nível regional, e pela coordenação e articulação técnica e política junto aos municípios de abrangência. (MATO GROSSO, 2013, p. 157)

Em 15 de março de 1994, foi instituído o Hemocentro Coordenador do Estado de Mato Grosso - Hemomat o qual, dez anos depois, teve a sua estrutura organizacional modificada recebendo nova denominação pela redação da Lei Complementar $\mathrm{n}^{\circ} 180$, de 13 de julho de 2004, passando a ser denominado MT - Hemocentro. (MATO GROSSO, 2016) Atualmente, a Hemorrede Estadual é composta por 1 Hemocentro Coordenador, 17 Unidades de Coletas e Transfusão e 29 agências Transfusionais, conforme a distribuição apresentada no quadro a seguir. 
Quadro 10 - Distribuição dos serviços de hematologia e hemoterapia do Sistema Único de Saúde nas macrorregiões, Mato Grosso, 2016

\begin{tabular}{|c|c|}
\hline Regiões & Quantidade/Unidades por nível de complexidade/municípios \\
\hline Norte Araguaia Carajás & Ol AT (São Félix do Araguaia) \\
\hline Sudoeste & O己 AT (Comodoro; Pontes e Lacerda) \\
\hline \multirow[t]{2}{*}{ Oeste } & Ol AT (São José dos Quatro Marcos) \\
\hline & O己 UCT (Cáceres; Mirassol D’Oeste) \\
\hline \multirow[t]{2}{*}{ Noroeste } & O己 AT (Aripuanã; Brasnorte) \\
\hline & OI UCT (Juína) \\
\hline \multirow[t]{2}{*}{ Médio Norte } & O3 AT (Campo Novo do Parecis; Sapezal) \\
\hline & O己 UCT (Barra do Bugres; Tangará da Serra) \\
\hline Alto Tapajós & OI UCT (Alta Floresta) \\
\hline Vale do Arinos & Ol UCT (Juara) \\
\hline Vale do Peixoto & O3 AT (Guarantã do Norte; Peixoto de Azevedo; Terra Nova do Norte) \\
\hline \multirow[t]{2}{*}{ Norte } & Ol AT (Marcelândia) \\
\hline & Ol UCT (Colíder) \\
\hline \multirow[t]{2}{*}{ Teles Pires } & O己 AT (Lucas do Rio Verde; Nova Mutum) \\
\hline & О२ UCT (Sinop; Sorriso) \\
\hline Centro Norte & O3 AT (Diamantino; Nobres; Nortelândia) \\
\hline \multirow[t]{3}{*}{ Baixada Cuiabana } & Ol HC [Cuiabá) \\
\hline & O3 AT (Cuiabá; Poconé; Várzea Grande) \\
\hline & Ol UCT (Cuiabá) \\
\hline \multirow[t]{2}{*}{ Baixo Araguaia } & O己 AT (Confresa; Vila Rica) \\
\hline & OI UCT (Porto Alegre do Norte) \\
\hline \multirow[t]{2}{*}{ Médio Araguaia } & Ol AT (Canarana) \\
\hline & Ol UCT (Água Boa) \\
\hline \multirow[t]{2}{*}{ Sul } & $\begin{array}{l}\text { O5 AT (Alto Araguaia; Campo Verde; Paranatinga; Poxoréo; } \\
\text { Rondonópolis) }\end{array}$ \\
\hline & O3 UCT (Jaciara, Primavera do Leste e Rondonópolis) \\
\hline \multirow{2}{*}{ Garças Araguaia } & Ol AT (Nova Xavantina) \\
\hline & Ol UCT (Barra do Garças) \\
\hline
\end{tabular}

Fonte: Mato Grosso (2016).

A cobertura hemoterápica do estado de Mato Grosso é realizada majoritariamente pela Hemorrede Pública (70,9\%) e complementada pela rede privada em 29,1\%. Quanto à atenção hematológica, o HC concentra o atendimento ambulatorial hematológico de todo o estado, incluindo exames diagnósticos 
e acompanhamento de portadores de Hemoglobinopatias, Coagulopatias, dentre outras doenças hematológicas. (MATO GROSSO, 2013)

\section{Considerações finais}

Como foi possível verificar, o desenho do curso, que origina o Projeto Planeja Sangue, apresenta uma proposta mista, envolvendo o ensino presencial e à distância e baseia-se numa estratégia ativa de aprendizagem, demandando um trabalho complexo para os cursistas, principalmente durante os momentos de dispersão, etapa na qual o apoio sistemático dos facilitadores torna-se essencial. A introdução do uso de metodologias de aprendizagem desta natureza tem sido adotada de forma gradativa nas ações de educação promovidas pela CGSH para a força de trabalho da Hemorrede Pública Nacional.

No caso aqui abordado, conforme já comentado, além da adequação à consecução dos objetivos traçados, evidenciada no processo avaliativo realizado ao final de 2015 pelo grupo de coordenação do projeto, a estratégia obteve excelente nível de adesão, bem como o reconhecimento dos cursistas, revelado por meio das avaliações orais, que ressaltaram sua importância para o desenvolvimento de habilidades para o trabalho coletivo. Além disto, a percepção sobre a oportunidade de discussão de problemas e soluções comuns junto aos pares da Hemorrede Estadual e demais segmentos envolvidos no processo de trabalho do curso a situa como uma oportunidade de ampliação do debate intrasetorial.

As dificuldades constatadas receberam tratamento, sendo estabelecidas soluções tal o empenho e experiência dos gestores, pontos focais e cursistas das hemorredes envolvidas, a expertise teórico metodológica da equipe do Instituto de Saúde Coletiva e o perfil profissional selecionado para a composição do grupo de mediadores e facilitadores das oficinas.

Observou-se a necessidade de desenvolvimento de parâmetros para a atenção hematológica de forma a instrumentalizar a análise da situação de saúde nessa perspectiva, a exemplo das referências já utilizadas no campo da atenção hemoterápica. Para tanto, encontra-se instituído grupo técnico para o desenvolvimento das referências ora citadas.

Uma limitação evidenciada, ao final do trabalho em 2015, foi a formatação final dos Planos Diretores pelas Hemorredes, demandando assim uma pro- 
posta já elaborada de acompanhamento e monitoramento, a ser adotada a partir de 2016, após o término do Momento Presencial 3, assegurando o apoio dos facilitadores até a conclusão efetiva dos Planos.

A continuidade do projeto está assegurada no planejamento da CGSH, de acordo com a disponibilidade futura de recursos para sua efetivação. Pretende-se, para o ano seguinte, em 2016, a oferta dos cursos de Planejamento e Gestão em outros sete estados (Rio de Janeiro, Mato Grosso do Sul, Piauí, Alagoas, Santa Catarina, Espírito Santo e Rio Grande do Sul).

Espera-se que as informações produzidas pelas Hemorredes, no Planeja Sangue, adquiram relevância como fonte de informação para a gestão da Política Nacional de Sangue e Hemoderivados e o aperfeiçoamento e fortalecimento das Hemorredes públicas estaduais.

\section{Referências}

AGÊNCIA NACIONAL DE VIGILÂNCIA SANITÁRIA. Resolução RDC nº 151, de 21 de agosto de 2001. Aprova o Regulamento Técnico sobre Níveis de Complexidade dos Serviços de Hemoterapia, que consta como anexo. Diário Oficial [da] República Federativa do Brasil, Poder Executivo, Brasília, DF, 22 ago. 2001. Seção 1, p. 29.

BAHIA. Lei $n^{\circ} 13.204$, de 11 de dezembro de 2014. Modifica a estrutura organizacional da Administração Pública do Poder Executivo Estadual e dá outras providências. Salvador, 2014.

BAHIA. Resolução CIB n ${ }^{\circ} 275$, de 15 de agosto de 2012. Aprova as regiões de saúde do Estado da Bahia e a instituição das Comissões Intergestores Regionais. Salvador, 2012a. Disponível em: <http://wwwl.saude.ba.gov.br/ mapa_bahia/docs/RESOLU\%C3\%87\%C3\%83O\%20275_15.08.2012_Aprova\%20 Regi\%C3\%B5es\%20de\%20Sa\%C3\%BAde\%20e\%20CIR.pdf >. Acesso em: 14 set. 2013.

BAHIA. Secretaria de Saúde do Estado da Bahia. Plano Estadual de Saúde da Bahia: 2012-2015. Salvador, 2012b.

BRANDÃO, I. C. A. et al. Análise da organização da rede de saúde da Paraíba a partir do modelo de regionalização. Revista Brasileira de Ciências da Saúde, Paraíba, v. 16, n. 3, p. 347-352, 2012.

BRASIL. Lei $n^{\circ} 10.205$, de 21 de março de 2001. Regulamenta o $§ 4^{\circ}$ do art. 199 da Constituição Federal, relativo à coleta, processamento, estocagem, distribuição e aplicação do sangue, seus componentes e derivados, 
estabelece o ordenamento institucional indispensável à execução adequada dessas atividades, e dá outras providências. Diário Oficial [da] República Federativa do Brasil, Poder Legislativo, Brasília, DF, 22 mar. 2001. Seção 1, p. 1. Disponível em <http://www.planalto.gov.br/ccivil_03/leis/leis_2001/110205. htm>. Acesso em: 10 nov. 2015.

BRASIL. Ministério da Saúde. Portaria $n^{\circ} 1.631$, de $1^{\circ}$ de outubro de 2015. Aprova critérios e parâmetros para o planejamento e programação de ações e serviços de saúde no âmbito do SUS. Diário Oficial [da] República Federativa, Brasília, DF, 2 out. 2015. Seção 1, p. 38.

BRASIL. Ministério da Saúde. Rede Interagencial de Informações para a Saúde. Indicadores de mortalidade. Brasília, DF, 2012. Disponível em: <http:// tabnet.datasus.gov.br/cgi/idb2012/c01b.htm>. Acesso em: 19 nov. 2016.

BRASIL. Ministério da Saúde. Secretaria de Atenção à Saúde. Departamento de Atenção Especializada. Gestão de hemocentros: relatos de práticas desenvolvidas no Brasil: I curso de especialização em gestão de hemocentros: resumos das monografias finais. Brasília, DF, 2010. 674 p. Disponível em: <http://bvsms.saude.gov.br/bvs/publicacoes/gestao_ hemocentros_praticas_brasil.pdf $>$. Acesso em: 10 mar. 2016.

BRASIL. Ministério da Saúde. Secretaria de Atenção à Saúde. Departamento de Atenção Especializada e Temática. Caderno de informação: sangue e hemoderivados: dados de 2014. 9. ed. Brasília, DF, 2015. 154 p. Disponível em: $<$ http://bvsms.saude.gov.br/bvs/publicacoes/caderno_informacao_sangue hemoderivados_dados_201_9ed.pdf >. Acesso em: 19 nov. 2016.

FUNDAÇÃO DE HEMATOLOGIA E HEMOTERAPIA DO ESTADO DA BAHIA. Plano diretor de sangue, componentes e derivados do estado da Bahia: 20162019. Salvador, 2016.

FUNDAÇÃO HEMOCENTRO DE PERNAMBUCO. Plano diretor de sangue, hemocomponentes e hemoderivados. Recife, 2016. No prelo.

FUNDAÇÃO HEMOCENTRO DE PERNAMBUCO. Recife, [200-]. Disponível em: <http://www.hemope.pe.gov.br/fund-interiorizacao.php >. Acesso em: 3 mar. 2016.

IBGE. Censo demográfico 2010: características da população e dos domicílios: resultados do universo. Rio de Janeiro, 2011.

IBGE. Estados @. Rio de Janeiro, [201-]. Disponível em: <http://www.ibge.gov. br/estadosat/perfil.php>. Acesso em: 19 nov. 2016. 
IBGE. Rio de Janeiro, [200-]. Disponível em: <http://cod.ibge.gov.br >. Acesso em: 10 mar. 2016.

LUCKESI, C. C. Avaliação da aprendizagem na escola: reelaborando conceitos e recriando a prática. Salvador: Malabares, 2003.

MATO GROSSO. Secretaria de Estado de Saúde. Hemocentro: quem somos. Cuiabá, [200-]. Disponível em: <http://www.saude.mt.gov.br/hemocentro/ pagina/70/quem-somos>. Acesso em: 10 mar. 2016.

MATO GROSSO. Secretaria de Estado de Saúde. Plano estadual de saúde de Mato Grosso: 2012-2015. Cuiabá, 2016. No prelo.

MATO GROSSO. Secretaria de Estado de Saúde. Superintendência de Políticas de Saúde. Plano estadual de saúde de MT: 2012-2015. Cuiabá, 2013.

PARAÍBA. Secretaria de Estado da Saúde. Gerência de Planejamento e Gestão. Sub-Gerência de Programação e Organização da Assistência. Núcleo de desenvolvimento das regiões de saúde. Plano diretor de regionalização da Paraíba. João Pessoa, 2008.

PERNAMBUCO. Secretaria Estadual de Saúde. Plano estadual de saúde: 20122015. Recife, 2012.

PERNAMBUCO. Secretaria de Saúde. Plano diretor de regionalização. Recife, 2011.

SANTOS, C. M. et al. Estudo de caso. In: SOUZA, M. K. B. et al. (Org.). Planeja Sangue: cursos em planejamento e gestão de sistema e serviços de saúde: guia para estudos. [S.l.]: Salvador, 2015. p. 127-132.

SOUZA, M. K. B. et al. (Org.). Planeja Sangue: cursos em planejamento e gestão de sistema e serviços de saúde: guia para estudos. [S.l.]: Salvador, 2015.

UNIVERSIDADE FEDERAL DA BAHIA. Instituto de Saúde Coletiva. Regimento do curso de graduação em Saúde Coletiva. Salvador, 2015. 